Interpretation of the depths of maximum of extensive air showers measured by the Pierre Auger Observatory

This article has been downloaded from IOPscience. Please scroll down to see the full text article.

JCAP02(2013)026

(http://iopscience.iop.org/1475-7516/2013/02/026)

View the table of contents for this issue, or go to the journal homepage for more

Download details:

IP Address: 146.139.228.12

The article was downloaded on 19/06/2013 at 21:43

Please note that terms and conditions apply. 


\title{
Interpretation of the depths of maximum of extensive air showers measured by the Pierre Auger Observatory
}

\section{The Pierre Auger collaboration}

\author{
E-mail: auger_spokespersons@fnal.gov
}

Received December 14, 2012

Accepted January 26, 2013

Published February 19, 2013

\begin{abstract}
To interpret the mean depth of cosmic ray air shower maximum and its dispersion, we parametrize those two observables as functions of the first two moments of the $\ln A$ distribution. We examine the goodness of this simple method through simulations of test mass distributions. The application of the parameterization to Pierre Auger Observatory data allows one to study the energy dependence of the mean $\ln A$ and of its variance under the assumption of selected hadronic interaction models. We discuss possible implications of these dependences in term of interaction models and astrophysical cosmic ray sources.
\end{abstract}

Keywords: ultra high energy cosmic rays, cosmic ray experiments 


\section{Contents}

1 Introduction 1

$2 \quad$ A method to interpret $\left\langle X_{\max }\right\rangle$ and $\sigma\left(X_{\max }\right) \quad 2$

3 Testing the method with simulation 3

4 Application to data $\quad 6$

5 Discussion $\quad 9$

6 Conclusions 11

A Parameterization of the shower mean depth and its fluctuations 12

$\begin{array}{lr}\text { The Pierre Auger collaboration } & 16\end{array}$

\section{Introduction}

The most commonly used shower observables for the study of the composition of Ultra High Energy Cosmic Rays (UHECR) are the mean value of the depth of shower maximum, $\left\langle X_{\max }\right\rangle$, and its dispersion, $\sigma\left(X_{\max }\right)$. Inferring the mass composition from these measurements is subject to some level of uncertainty. This is because their conversion to mass relies on the use of shower simulation codes which include the assumption of a hadronic interaction model. The various interaction models [1] have in common the ability to fit lower energy accelerator data. However, different physical assumptions are used to extrapolate these low energy interaction properties to higher energies. Consequently they provide different expectations for $\left\langle X_{\max }\right\rangle$ and $\sigma\left(X_{\max }\right)$. The first aim of this paper is to discuss how the mean value of the depth of shower maximum and its dispersion can be used to interpret mass composition even in the presence of uncertainties in the hadronic interaction modeling.

Furthermore, we discuss the different roles of the two observables, $\left\langle X_{\max }\right\rangle$ and $\sigma\left(X_{\max }\right)$, with respect to mass composition. In the interpretation of data they are often used as different, and independent, aspects of the same phenomenon. However it is not true to say that both parameters reflect the cosmic ray composition to the same extent. According to the superposition model [2] $\left\langle X_{\max }\right\rangle$ is linear in $\langle\ln A\rangle$ and therefore it actually measures mass composition for both pure and mixed compositions. But, we will show that the behaviour of $\sigma\left(X_{\max }\right)$ is more complex to interpret as there is no one-to-one correspondence between its value and a given mean log mass. Only in the case of pure composition is this correspondence unique.

In this paper we refine the analysis method originally proposed by Linsley [3, 4] and apply it to the Auger data. The Pierre Auger Collaboration has published results on the mean and dispersion of the $X_{\max }$ distribution at energies above $10^{18} \mathrm{eV}[5,6]$. In this work we apply the proposed method to convert those observables to the first moments of the log mass distribution, namely $\langle\ln A\rangle$ and $\sigma_{\ln A}^{2}$.

The paper is organized as follows. In section 2 we discuss the parameterization for $\left\langle X_{\max }\right\rangle$ and $\sigma\left(X_{\max }\right)$. In section 3 we test the method with shower simulations assuming 
different mass distributions. Section 4 describes the application of the method to data. The discussion of the results and the conclusions follow in sections 5 and 6 respectively. The details of the parameterization and the best fit values for the hadronic interaction models are summarized in appendix A.

\section{A method to interpret $\left\langle X_{\max }\right\rangle$ and $\sigma\left(X_{\max }\right)$}

The interpretation of $\left\langle X_{\max }\right\rangle$ and $\sigma\left(X_{\max }\right)$ can be simplified by making use of an analysis method based on the generalized Heitler model of extensive air showers [7]. In this context $\left\langle X_{\max }\right\rangle$ is a linear function of the logarithm of the shower energy per nucleon:

$$
\left\langle X_{\max }\right\rangle=X_{0}+D \log _{10}\left(\frac{E}{E_{0} A}\right),
$$

where $X_{0}$ is the mean depth of proton showers at energy $E_{0}$ and $D$ is the elongation rate [810], i.e., the change of $\left\langle X_{\max }\right\rangle$ per decade of energy. The High Energy hadronic interaction models used in this work are EPOS 1.99 [11], Sibyll 2.1 [12], QGSJet 01 [13] and QGSJet II [14]. Simulated data show that eq. (2.1) gives a fair description of EPOS and Sibyll results in the full range of interest for this work, $10^{18}$ to $10^{20} \mathrm{eV}$, but does not reproduce accurately QGSJet models. For this reason we generalize the original representation as:

$$
\left\langle X_{\max }\right\rangle=X_{0}+D \log _{10}\left(\frac{E}{E_{0} A}\right)+\xi \ln A+\delta \ln A \log _{10}\left(\frac{E}{E_{0}}\right),
$$

where the parameters $\xi$ and $\delta$ are expected to be zero if the model predictions are compatible with the superposition result (2.1).

For nuclei of the same mass $A$ one expects the shower maximum to be on average:

$$
\left\langle X_{\max }\right\rangle=\left\langle X_{\max }\right\rangle_{p}+f_{E} \ln A,
$$

and its dispersion to be only influenced by shower-to-shower fluctuations:

$$
\sigma^{2}\left(X_{\max }\right)=\sigma_{\mathrm{sh}}^{2}(\ln A) .
$$

Here $\left\langle X_{\max }\right\rangle_{p}$ denotes the mean depth at maximum of proton showers, as obtained from either eq. (2.1) or (2.2), and $\sigma_{\mathrm{sh}}^{2}(\ln A)$ is the $X_{\max }$ variance for mass $A, \sigma_{\mathrm{sh}}^{2}(\ln A)=\sigma^{2}\left(X_{\max } \mid \ln A\right)$. The energy dependent parameter $f_{E}$ appearing in (2.3) is:

$$
f_{E}=\xi-\frac{D}{\ln 10}+\delta \log _{10}\left(\frac{E}{E_{0}}\right) .
$$

The values of the parameters $X_{0}, D, \xi, \delta$ depend on the specific hadronic interaction model. In this work they are obtained from CONEX [15] shower simulations as described in appendix A.

In the case of a mixed composition at the top of the atmosphere, the mean and variance of $X_{\max }$ depend on the $\ln A$ distribution. There are two independent sources of fluctuations: the intrinsic shower-to-shower fluctuations and the $\ln A$ dispersion arising from the mass distribution. The first term gives rise to $\left\langle\sigma_{\mathrm{sh}}^{2}\right\rangle$, the average variance of $X_{\max }$ weighted according to the $\ln A$ distribution. The second contribution can be written as $\left(\frac{d\left\langle X_{\max }\right\rangle}{d \ln A}\right)^{2} \sigma_{\ln A}^{2}$ where $\sigma_{\ln A}^{2}$ is the variance of the $\ln A$ distribution. We can finally write for the two profile observables:

$$
\begin{aligned}
\left\langle X_{\max }\right\rangle & =\left\langle X_{\max }\right\rangle_{p}+f_{E}\langle\ln A\rangle \\
\sigma^{2}\left(X_{\max }\right) & =\left\langle\sigma_{\mathrm{sh}}^{2}\right\rangle+f_{E}^{2} \sigma_{\ln A}^{2} .
\end{aligned}
$$


The two equations depend on energy through the parameters but also via $\left\langle\sigma_{\mathrm{sh}}^{2}\right\rangle$ and the possible dependence of the two moments of the $\ln A$ distribution.

To obtain an explicit expression for $\left\langle\sigma_{\mathrm{sh}}^{2}\right\rangle$ we need a parameterization for $\sigma_{\mathrm{sh}}^{2}(\ln A)$. We assume a quadratic law in $\ln A$ :

$$
\sigma_{\mathrm{sh}}^{2}(\ln A)=\sigma_{p}^{2}\left[1+a \ln A+b(\ln A)^{2}\right],
$$

where $\sigma_{p}^{2}$ is the $X_{\max }$ variance for proton showers. The evolution of $\sigma_{\mathrm{sh}}^{2}(\ln A)$ with energy is included in $\sigma_{p}^{2}$ and the parameter $a$ :

$$
\sigma_{p}^{2}=p_{0}+p_{1} \log _{10}\left(\frac{E}{E_{0}}\right)+p_{2}\left[\log _{10}\left(\frac{E}{E_{0}}\right)\right]^{2} \quad \text { and } \quad a=a_{0}+a_{1} \log _{10}\left(\frac{E}{E_{0}}\right) .
$$

The parameters $p_{0}, p_{1}, p_{2}, a_{0}, a_{1}, b$ depend on hadronic interactions: the values used in the paper are given in appendix $\mathrm{A}$.

Using measurements of $\left\langle X_{\max }\right\rangle$ and $\sigma\left(X_{\max }\right)$, equations (2.6) and (2.7) can be inverted to get the first two moments of the $\ln A$ distribution. From eq. (2.6) one gets:

$$
\langle\ln A\rangle=\frac{\left\langle X_{\max }\right\rangle-\left\langle X_{\max }\right\rangle_{p}}{f_{E}} .
$$

Averaging eq. (2.8) on $\ln A$ one obtains:

$$
\left\langle\sigma_{\mathrm{sh}}^{2}\right\rangle=\sigma_{p}^{2}\left[1+a\langle\ln A\rangle+b\left\langle(\ln A)^{2}\right\rangle\right] .
$$

Substituting in eq. (2.7) we get:

$$
\sigma^{2}\left(X_{\max }\right)=\sigma_{p}^{2}\left[1+a\langle\ln A\rangle+b\left\langle(\ln A)^{2}\right\rangle\right]+f_{E}^{2} \sigma_{\ln A}^{2} .
$$

But by definition $\left\langle(\ln A)^{2}\right\rangle=\sigma_{\ln A}^{2}+\langle\ln A\rangle^{2}$. Solving in $\sigma_{\ln A}^{2}$ one finally obtains:

$$
\sigma_{\ln A}^{2}=\frac{\sigma^{2}\left(X_{\max }\right)-\sigma_{\mathrm{sh}}^{2}(\langle\ln A\rangle)}{b \sigma_{p}^{2}+f_{E}^{2}} .
$$

Equations (2.10) and (2.13) are the key tools used throughout this work for interpreting Pierre Auger Observatory data in terms of mass composition and assessing the validity of available hadronic interaction models.

\section{$3 \quad$ Testing the method with simulation}

Equations (2.6) and (2.7) can be tested with simulations. They contain parameters depending on the hadronic interaction properties and on the mass distribution of nuclei. The mass distribution of nuclei refers to those nuclei hitting the Earth's atmosphere: it does not matter what is the source of the mass dispersion, either a mixed composition at injection or the dispersion caused by propagation. So, in order to test the method we will simply use different test distributions of the masses at the top of the atmosphere.

For this purpose we have chosen three different mass distributions:

1. A distribution uniform in $\ln A$ from $\ln (1)$ to $\ln (56)$ and independent of energy. The values of $\langle\ln A\rangle$ and $\sigma_{\ln A}$ are respectively 2.01 and 1.16 . 

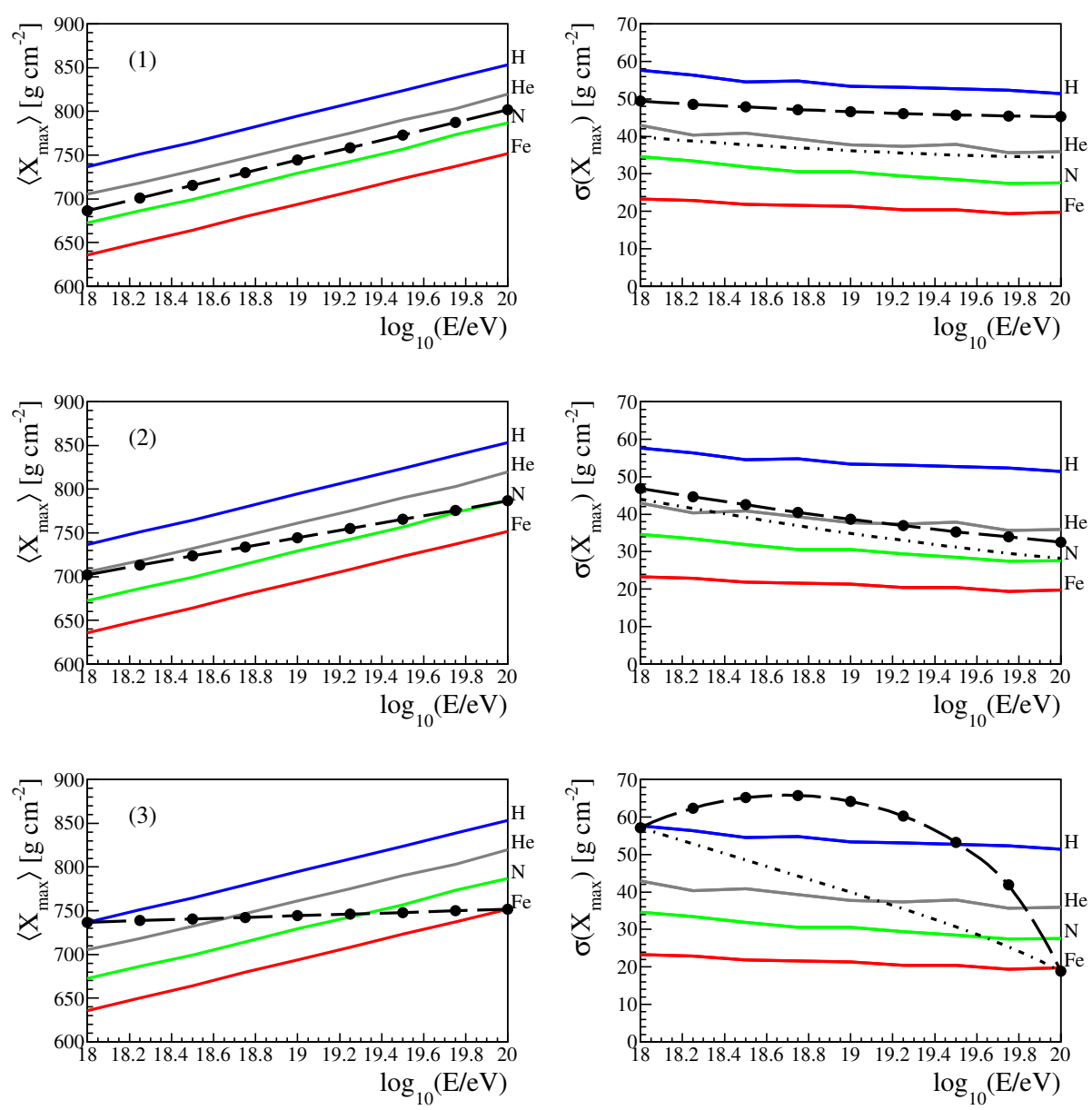

Figure 1. $\left\langle X_{\max }\right\rangle$ and $\sigma\left(X_{\max }\right)$ as a function of $\log _{10}(E / \mathrm{eV})$ for three different mass distribution hypotheses (see text). Full circles are calculated from the resulting $X_{\max }$ distributions from the CONEX simulations. Sibyll 2.1 has been chosen for hadronic interactions. The dashed lines show equations (2.6) for $\left\langle X_{\max }\right\rangle$ and (2.7) for $\sigma\left(X_{\max }\right)$. The dot-dashed line refers to the contribution of the first term in (2.7).

2. A Gaussian $\ln A$ distribution with $\langle\ln A\rangle$ increasing linearly with $\log E$ from $\ln (4)$ at $10^{18} \mathrm{eV}$ to $\ln (14)$ at $10^{20} \mathrm{eV}$ and $\sigma_{\ln A}=0.75$ independent of energy. The Gaussian is truncated to less than 2 sigmas to avoid unphysical mass values. In this case the $\ln A$ dispersion is fixed and equal to 0.66 but $\langle\ln A\rangle$ varies with energy.

3. Two masses, $\mathrm{H}$ and $\mathrm{Fe}$, with proton fraction $H /(H+F e)$ decreasing linearly with $\log E$ from 1 at $10^{18} \mathrm{eV}$ to 0 at $10^{20} \mathrm{eV}$. In this case, both $\langle\ln A\rangle$ and $\sigma_{\ln A}$ vary with energy.

Figure 1 shows the result of the test for the three mass distribution hypotheses. To generate the $X_{\max }$ distributions we have used CONEX [15] showers with Sibyll 2.1 [12] as the hadronic interaction model. These distributions do not include detector effects. For each test mass hypothesis, the mean and RMS are retrieved from the resulting $X_{\max }$ distribution obtained from the simulations. These are shown as full circles, $\left\langle X_{\max }\right\rangle$ and $\sigma\left(X_{\max }\right)$ in left and right panels respectively. The dashed lines are calculated using equations (2.6) and (2.7) for the three different mass hypotheses by using only the first two moments $\langle\ln A\rangle$ and $\sigma_{\ln A}$. 

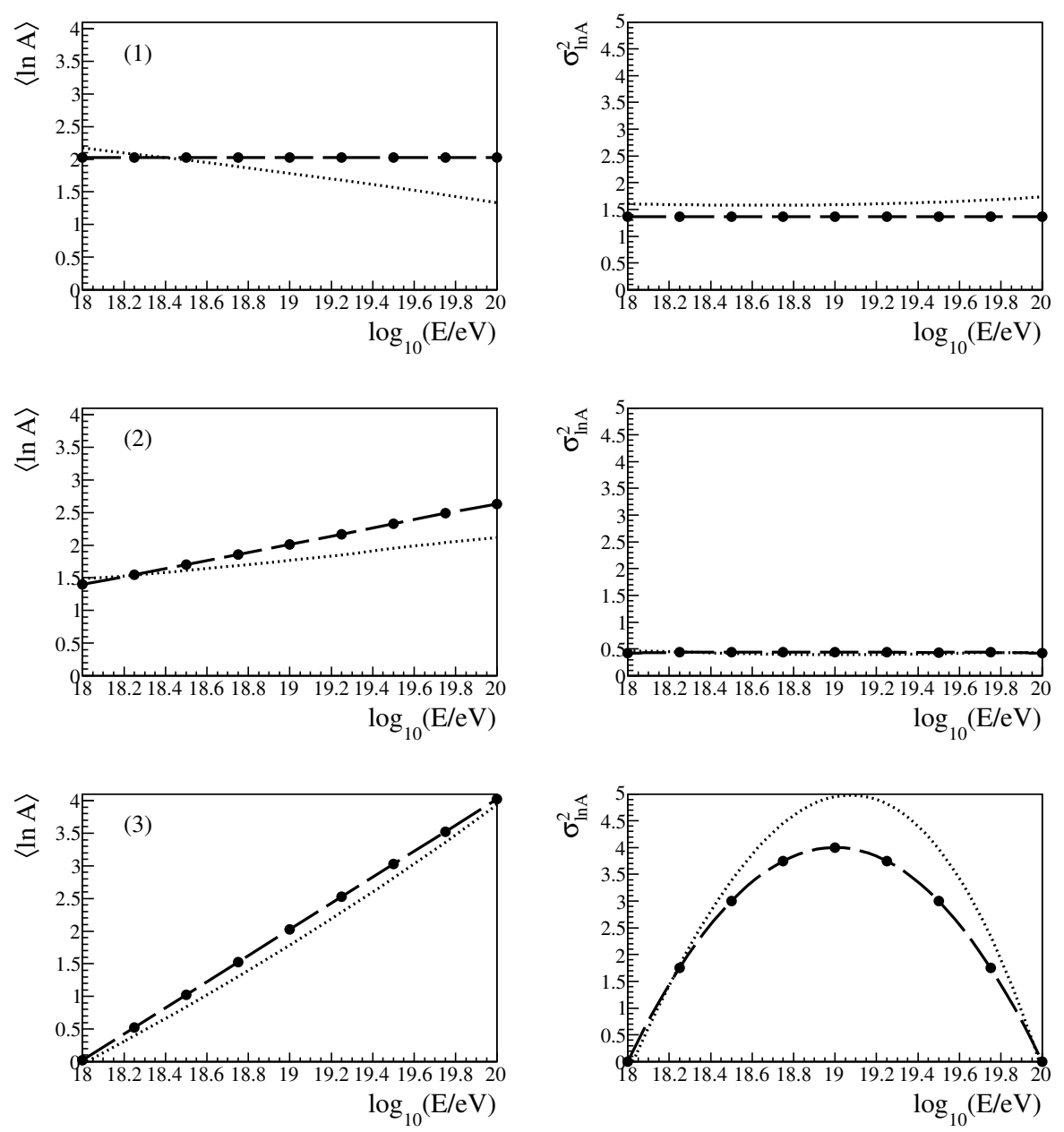

Figure 2. $\langle\ln A\rangle$ and $\sigma_{\ln A}^{2}$ as a function of $\log _{10}(E / e V)$ for three different mass distribution hypotheses. Sibyll 2.1 is the hadronic interaction model. Full circles refer to the values obtained directly from the input mass distributions. The dashed lines show $\langle\ln A\rangle$ and $\sigma_{\ln A}^{2}$ calculated using equations (2.10) and (2.13). The dotted lines refer to the calculation of the same variables using the parameterization for QGSJet II in (2.10) and (2.13).

One can see that, despite the simple assumptions made, good agreement is achieved for all the three mass distributions. The dot-dashed line refers to the contribution of the first term in eq. (2.7). The comparison between the two lines (dashed vs. dot-dashed) highlights how different the interpretation of $\sigma\left(X_{\max }\right)$ data can be if one does not take into account the mass dispersion term.

The inverse equations (2.10) and (2.13) have also been tested using Monte Carlo simulation. In this case $\langle\ln A\rangle$ and $\sigma_{\ln A}^{2}$ have been obtained as a function of $\log _{10}(E / \mathrm{eV})$ directly from the input mass distributions. These values are shown as full circles in figure 2. The $\left\langle X_{\max }\right\rangle$ and $\sigma\left(X_{\max }\right)$ retrieved from the corresponding $X_{\max }$ distributions are used in equations (2.10) and (2.13) to get $\langle\ln A\rangle$ and $\sigma_{\ln A}^{2}$. These are shown in figure 2 as dashed lines. Also in this case, the comparison is quite successful.

The simulated data sample can also be used to estimate the systematic uncertainty in the calculation of the moments of the $X_{\max }(\ln A)$ distribution induced by the missing knowl- 

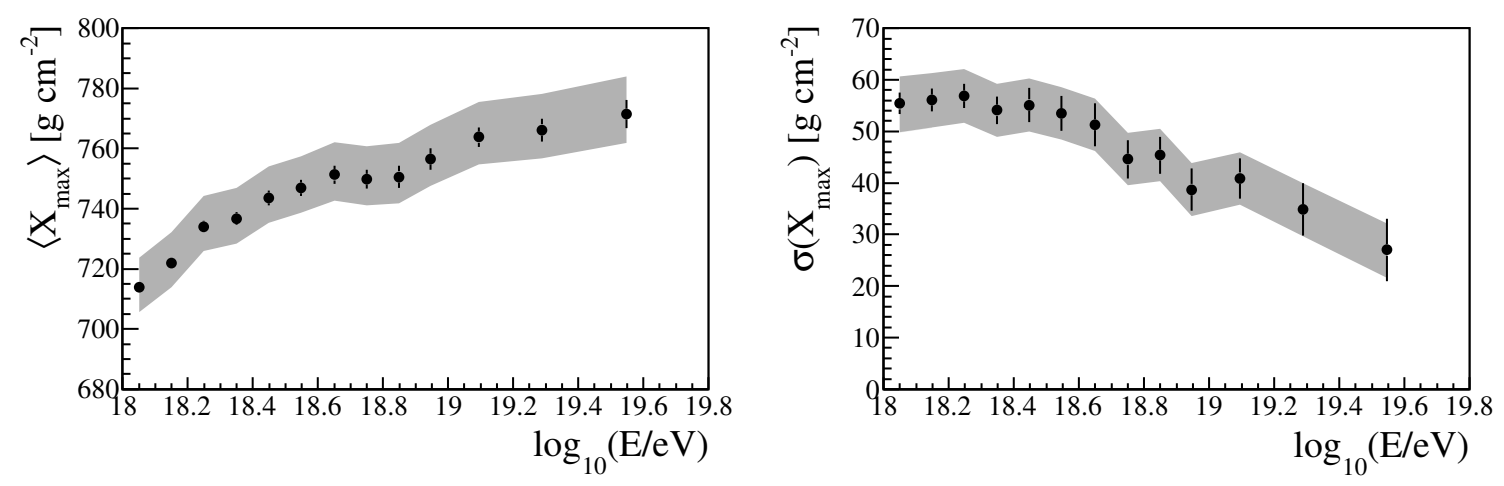

Figure 3. $\left\langle X_{\max }\right\rangle$ (left) and $\sigma\left(X_{\max }\right)$ (right) as a function of $\log _{10}(E / \mathrm{eV})$ from Pierre Auger Observatory data $[6,22]$. Data (full circles) are shown with statistical errors. Systematic uncertainties are represented as bands.

edge of the hadronic interaction mechanism. This study is pursued using simulated showers generated with a given model together with parameters of another model in equations (2.6) and (2.7) for the profile variables, and (2.10) and (2.13) for the log mass variables. An example of this procedure is shown in figure 2 where the dotted lines show the calculation with the parameters of QGSJet II and the full circles refer to data simulated with Sibyll 2.1. As a summary of these cross-model checks, we find mean absolute deviations of 4 to $27 \mathrm{~g} \mathrm{~cm}^{-2}$ for $\left\langle X_{\max }\right\rangle$ and 1 to $5.4 \mathrm{~g} \mathrm{~cm}^{-2}$ for $\sigma\left(X_{\max }\right)$, where the maximum deviations are obtained crossing EPOS with QGSjetII. The same study done for the moments of the log mass distribution gives mean absolute deviations of 0.2 to 1.2 for $\langle\ln A\rangle$ and 0.02 to 0.5 for $\sigma_{\ln A}^{2}$. In this case the maximum values refer to EPOS vs. QGSJet 01 for the first moment and QGSJet II vs. QGSJet 01 for the second.

\section{Application to data}

At ultra-high energies, shower development can be directly measured using fluorescence and Cherenkov light profiles. Mean $X_{\max }$ data as a function of energy are available from Fly's Eye [16], HiRes [17, 18], Auger [5], Yakutsk [19] and Telescope Array [20]. $\left\langle X_{\max }\right\rangle$ data were complemented with fluctuation measurements as early as 1980s (see e.g. [21] and references therein) but only recently have precise optical detector measurements become available $[5$, $18,19]$.

The Pierre Auger Collaboration has published results on the mean and dispersion of the $X_{\max }$ distribution at energies above $10^{18} \mathrm{eV}$ [5]. Here we apply the method presented in this work to an updated dataset available in $[6,22]$. These data are shown in figure 3.

In the Auger analysis [5], the events are selected using fiducial volume cuts based on the shower geometry. This ensures that the viewable $X_{\max }$ range for each shower is large enough to accommodate the full $X_{\max }$ distribution. Also, the detector resolution is accounted for by subtracting in quadrature its contribution to the measured dispersion. This allows the direct conversion to the moments of the $\ln A$ distribution using equations (2.10) and (2.13) without the need of more complex treatment, such as is required in the presence of acceptance biases [23, 24]. 

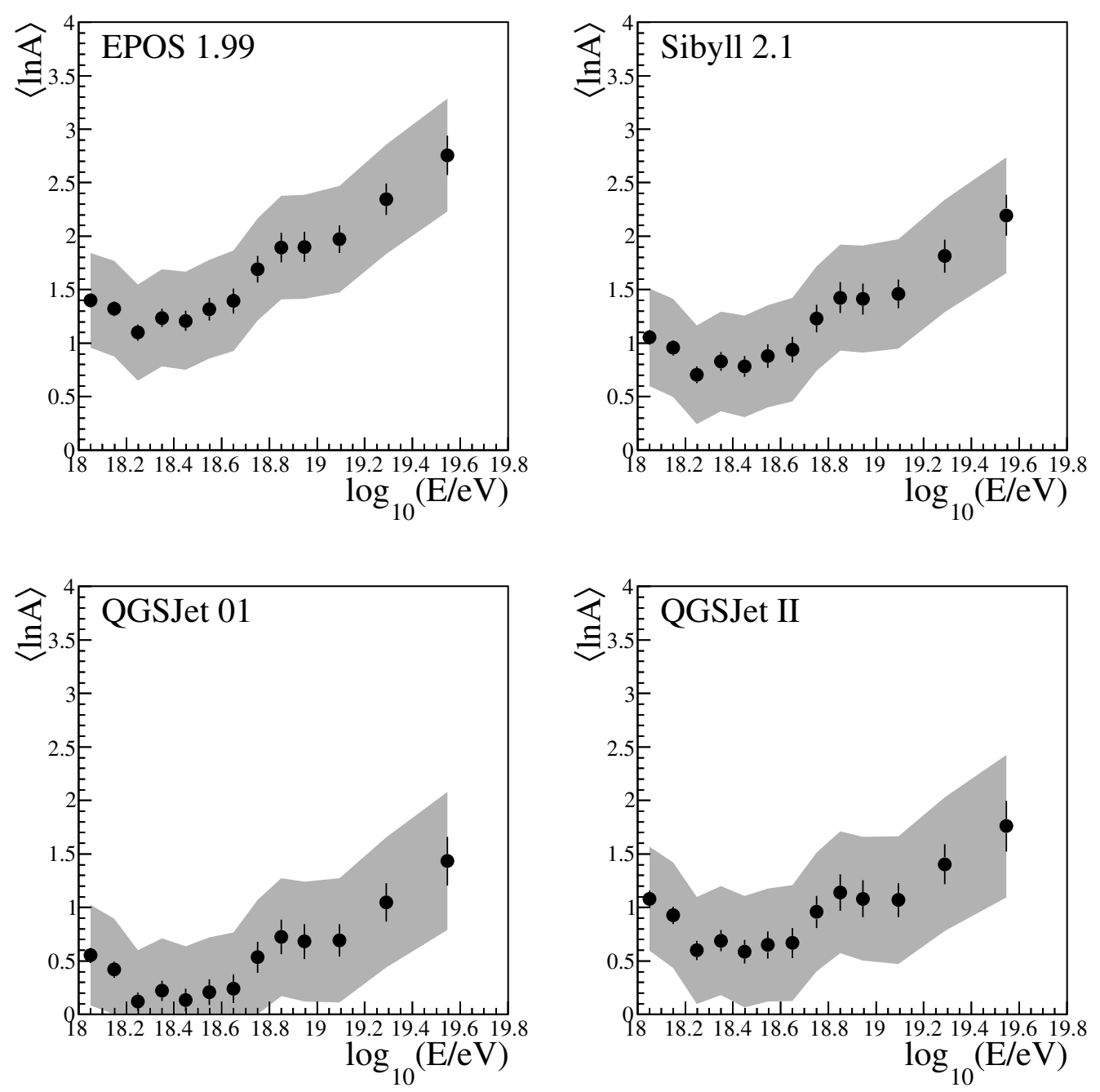

Figure 4. $\langle\ln A\rangle$ as a function of $\log _{10}(E / \mathrm{eV})$ obtained from Auger data [22] are shown as full circles for different hadronic interaction models. Error bars show statistical errors. The shaded areas refer to systematic uncertainties obtained by summing in quadrature the systematic uncertainties on $\left\langle X_{\max }\right\rangle$ and $\sigma\left(X_{\max }\right)$ data points and on the FD energy scale.

The moments of the $\log$ mass distribution, $\langle\ln A\rangle$ and $\sigma_{\ln A}^{2}$, as obtained using equations (2.10) and (2.13), are shown (full circles) as a function of $\log _{10}(E / \mathrm{eV})$ in figures 4 and 5 respectively. Error bars show the statistical errors obtained from the propagation of data errors and the errors of the fitted parameters. Shaded bands are the systematic uncertainties obtained by summing in quadrature the different individual contributions. The systematic uncertainties on $\left\langle X_{\max }\right\rangle$ and $\sigma\left(X_{\max }\right)$ data points have different sources: calibration, atmospheric conditions, reconstruction and event selection [5]. Another source of systematics is related to the uncertainty of the FD energy scale [25], $22 \%$, which induces an uncertainty in $\langle\ln A\rangle$ and $\sigma_{\ln A}^{2}$ via the parameters of the models. All these uncertainties contribute approximately at the same level and independently of energy. The figures show the results for the moments of the log mass distribution for EPOS 1.99 [11], Sibyll 2.1 [12], QGSJet 01 [13] and QGSJet II [14]. 

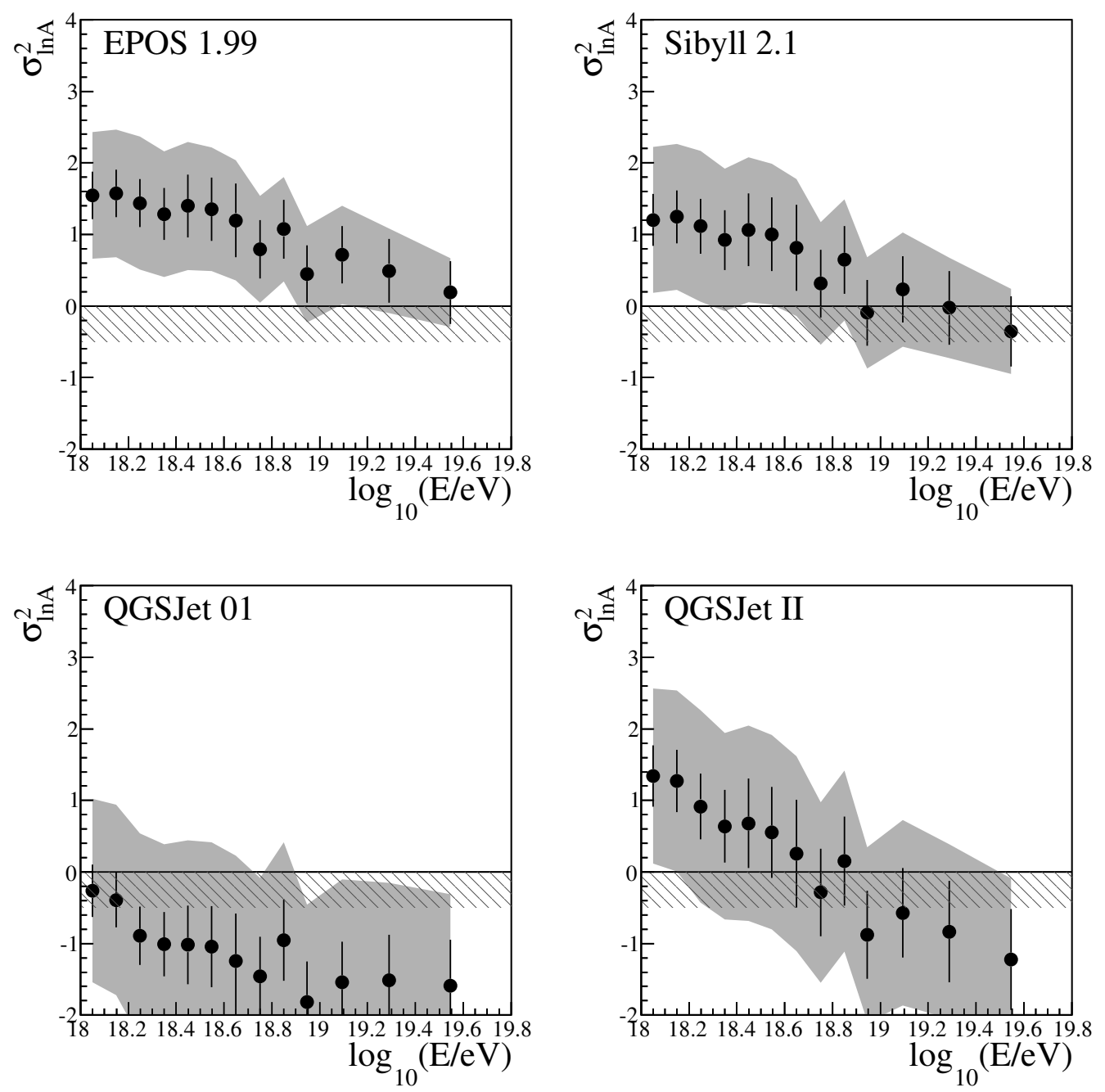

Figure 5. $\sigma_{\ln A}^{2}$ as a function of $\log _{10}(E / \mathrm{eV})$ obtained from Auger data [22] are shown as full circles for different hadronic interaction models. Error bars show statistical errors. The shaded area refers to systematic uncertainties as in figure 4. The lower limit of allowed $\sigma_{\ln A}^{2}$ is shown by the exclusion line. The upper limit (4.05) is just above the maximum of the vertical axis.

Despite the uncertainties and the different mass offsets of the models, the overall features are similar in all the cases. So far as the energy dependence is concerned, the data imply an increasing $\langle\ln A\rangle$ above $10^{18.3} \mathrm{eV}$ from light to intermediate masses and a decreasing $\sigma_{\ln A}^{2}$ over the whole energy range.

Looking more specifically to the different hadronic models we notice a slight change in the log mass scale. The highest masses are obtained for EPOS 1.99. Sibyll 2.1 and QGSJet II show intermediate values, whereas the lowest masses are obtained for QGSJet 01. In particular at $\log _{10}(E / \mathrm{eV})=18.25$ the mean $\log$ mass, $\langle\ln A\rangle$, is $1.10,0.70,0.60$ and 0.12 respectively for EPOS 1.99, Sibyll 2.1, QGSJet II and QGSJet 01 with statistical errors of about 0.08 and systematic uncertainty of about 0.6. The Pierre Auger Collaboration has recently published the measurement of the proton-air cross section for the energy interval $10^{18}$ to $10^{18.5} \mathrm{eV}$ [26]. That measurement is done using the showers with $X_{\max } \geq 768 \mathrm{~g} \mathrm{~cm}^{-2}$, 
corresponding to $20 \%$ of the total $X_{\max }$ distribution. Even in the most unfavourable case, (the $\langle\ln A\rangle$ and $\sigma_{\ln A}^{2}$ predicted by EPOS), one finds that several realizations obtained from the allowed $\langle\ln A\rangle$ and $\sigma_{\ln A}^{2}$ have enough protons in the most deeply penetrating showers to fulfill the selection criteria adopted in the Auger analysis.

Whereas $\langle\ln A\rangle$ always has valid values (apart a small region which crosses $\langle\ln A\rangle=0$ for QGSJet 01), there are wide energy intervals where $\sigma_{\ln A}^{2}$ is negative. Considering eq. (2.13) one can see that these values occur for energies where the shower fluctuations corresponding to the mean $\log$ mass exceed the measured $X_{\max }$ fluctuations. Figure 5 shows that $\sigma_{\ln A}^{2}$ data points are within the allowed physical region only for EPOS 1.99 and Sibyll 2.1. They are partly outside for QGSJet II, and completely outside for QGSJet 01. However the current systematic uncertainties do not allow one to establish stringent tests to the models.

The method presented in this work shows that the Pierre Auger Observatory data can confront hadronic physics models provided that future developments in the shower data analysis reduce systematics. By shrinking the shaded bands in figure 5 it will be possible to constrain those models.

\section{Discussion}

The importance of the combined study of the mean values and fluctuations of mass dependent observables has been addressed by several authors [3, 4, 21, 27, 28]. In particular, Linsley [4] showed that a combined analysis of the mean and the variance of $\ln A$ can provide a useful representation of the mass transition (if any) to be found in shower profile data. In fact, possible transitions are constrained to a limited region of the $\left(\langle\ln A\rangle, \sigma_{\ln A}^{2}\right)$ plane. More recently a similar study using the $\left\langle X_{\max }\right\rangle-\sigma\left(X_{\max }\right)$ correlation $^{1}$ reached a similar conclusion [29].

Converting $X_{\max }$ data to $\ln A$ variables, as described in section 2, one can plot Pierre Auger Observatory data in the $\left(\langle\ln A\rangle, \sigma_{\ln A}^{2}\right)$ plane. Since this procedure depends on the hadronic model, one gets a plot for each model as shown in figure 6. Data points are shown as full circles with size increasing in proportion to $\log E$. The error bars are tilted because of correlations arising from equations (2.10) and (2.13) and represent the principal axes of the statistical error ellipses. The solid lines show the systematic uncertainties. The same figure shows the region allowed for mass compositions. The contour of this region (gray thick line) is generated by mixing neighbouring nuclei in the lower edge and extreme nuclei (protons and iron) in the upper edge. Each of these mixings is an arch shaped line in the $(\langle\ln A\rangle$, $\left.\sigma_{\ln A}^{2}\right)$ plane.

Figure 6 shows that the Auger data lie outside the allowed boundaries for part of the energy range in some of the models. As noted previously, systematic uncertainties are still large and thus prevent us from more definite conclusions. However the energy evolution is common to all models suggesting that the average mass increases with decreasing log mass dispersion. This behaviour might imply astrophysical consequences.

In fact there are only a few possibilities for extragalactic source models to produce compositions with small log mass dispersion at the Earth. Protons can traverse their path from sources to the Earth without mass dispersion, but this case is excluded by Pierre Auger Observatory data at the highest energies.

Nuclei originating from nearby sources $(\lesssim 100 \mathrm{Mpc})$ might be detected with small mass dispersion. For these sources, propagation does not degrade mass and energy so the spectrum

\footnotetext{
${ }^{1}$ In this case the dependence on hadronic models has been accounted for by subtracting the corresponding observables predicted by the models for iron.
} 

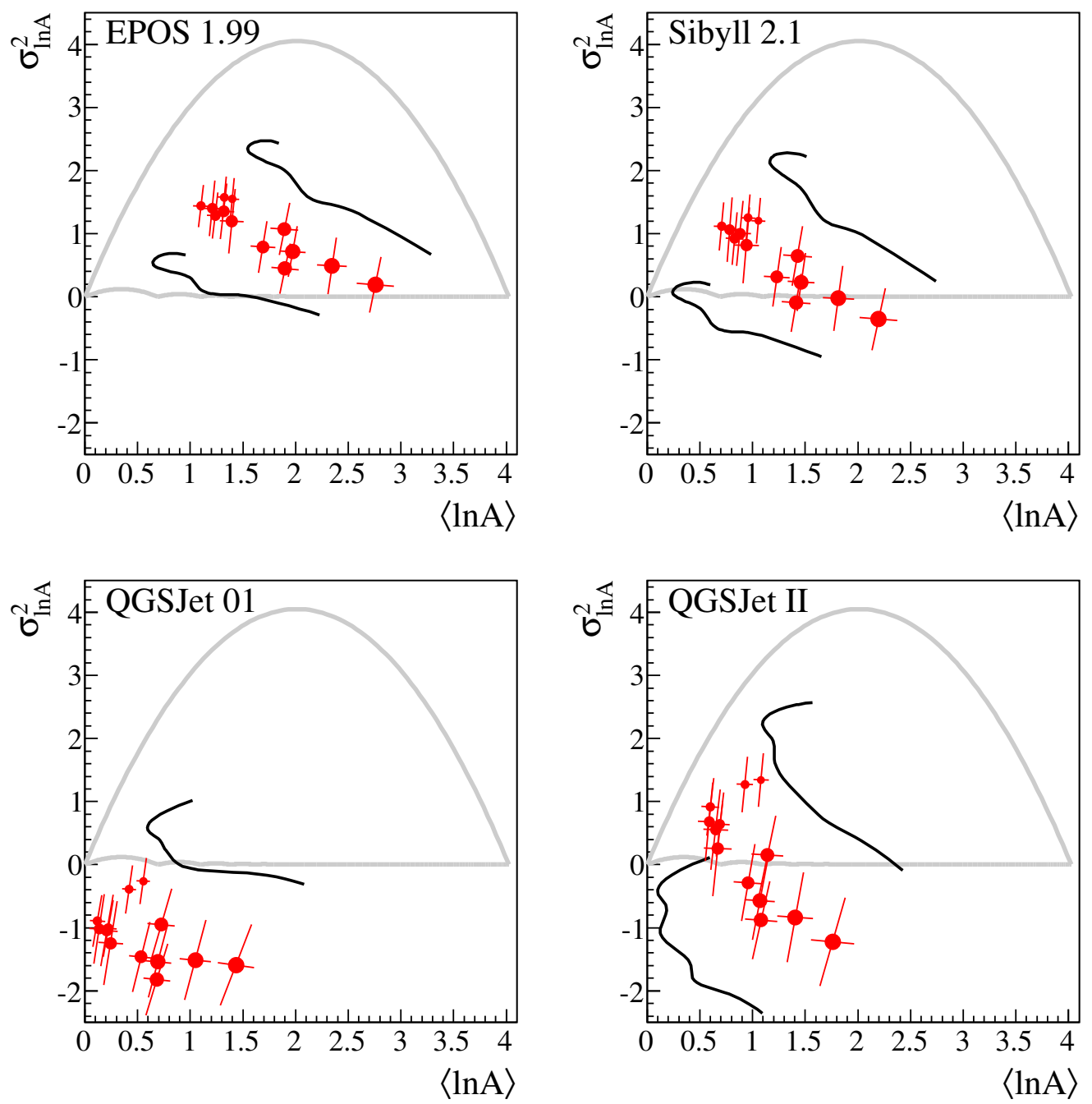

Figure 6. Pierre Auger data in the $\left(\langle\ln A\rangle, \sigma_{\ln A}^{2}\right)$ plane for different hadronic interaction models. Data points are shown as full circles with statistical errors. The marker sizes increase with the logarithm of the energy. Systematic uncertainties are shown as solid lines. The gray thick line shows the contour of the $\langle\ln A\rangle$ and $\sigma_{\ln A}^{2}$ values allowed for nuclear compositions.

and composition reflect closely their values at injection. But, if sources are distributed uniformly, distant sources induce natural mass dispersions. Small $\ln A$ dispersions are possible only when there is small observed mass mixing so that, at each energy, only nuclei with a small spread in masses are present. This corresponds to the low- $\sigma_{\ln A}^{2}$ edge of the contour of the allowed region in the $\left(\langle\ln A\rangle, \sigma_{\ln A}^{2}\right)$ plane.

Protons originating by the photo-disintegration of nuclei are the main source of mass dispersion because they populate each energy region. The possible end of the injection spectrum based on a rigidity-dependent mechanism can reduce the proton component at high energies, thus producing a reduction of the mass dispersion at the highest energies. A complete study of source models under several hypotheses is required to study all the source parameters that limit the mass dispersion in the propagation of extragalactic cosmic rays. Recent studies, see e.g. [30, 31], based on the assumption of a uniform source distribution, 
have shown that the Auger composition results, when combined with the energy spectrum, require hard injection spectra (i.e. index $<2$ ) with low energy cutoffs and the possible presence of local sources.

\section{Conclusions}

In this work we presented a method for interpreting $\left\langle X_{\max }\right\rangle$ and $\sigma\left(X_{\max }\right)$ in terms of mass composition. The method is based on an extension of the Heitler model of extensive air showers. The parameterization given in equations (2.6) and (2.7) expresses those two profile observables as a linear combination of the first two moments of the log mass distribution, $\langle\ln A\rangle$ and $\sigma_{\ln A}^{2}$, and of the mean shower fluctuations.

We first note that the method provides an effective key to the interpretation of data. The energy dependences of $\left\langle X_{\max }\right\rangle$ and $\sigma\left(X_{\max }\right)$ are sometimes considered as different expressions of the same physical features, e.g. an increase or decrease of the mean log mass. However their different meanings can be easily understood by looking at the dependence on the mass variables. At a fixed energy $\left\langle X_{\max }\right\rangle$ is only function of $\langle\ln A\rangle$; therefore, it only carries information of the average composition. However, $\sigma\left(X_{\max }\right)$ cannot be interpreted as a measure of the average composition since it is also affected by the log mass dispersion. Similarly, the inference of hadronic interaction properties from $\sigma\left(X_{\max }\right)$ can be wrong unless the mass dispersion term $\left(\propto \sigma_{\ln A}^{2}\right)$ is negligible. The parameter $\sigma_{\ln A}$ represents the dispersion of the masses as they hit the Earth atmosphere. It reflects not only the spread of nuclear masses at the sources but also the modifications that occur during their propagation to the Earth.

The method has been succesfully tested, with the simulation of different mass distributions in the energy interval from $10^{18}$ to $10^{20} \mathrm{eV}$ showing the robustness of the parameterization. We have applied the method to the Pierre Auger Observatory $X_{\max }$ data to get the first two moments of the $\ln A$ distribution. The outcome relies on the choice of a hadronic interaction model to set the parameters and the appropriate shower fluctuations. Four models have been used, EPOS 1.99, Sibyll 2.1, QGSJet 01 and QGSJet II, and the corresponding moments of the log mass distribution have been obtained as a function of energy. Despite the differences in the chosen models, the overall features are quite similar. In particular we find an increasing $\langle\ln A\rangle$ above $10^{18.3} \mathrm{eV}$ from light to intermediate masses and a decreasing $\sigma_{\ln A}^{2}$ over the whole energy range, while the mean log mass scale changes with hadronic models.

The results presented in this paper show the capability of the method to infer important features of the mass distribution of the UHECR nuclei. This is a remarkable outcome with respect to the study of source scenarios and propagation. In fact we do not only access the average mass, but also the mass dispersion. While a pure proton beam at the sources is not changed by propagation, nuclei should increase the mass dispersion in their path towards the Earth. The Auger results seem to imply either close-by sources or hard spectral indices, if the energy evolution of the present hadronic interaction models can be trusted.

The proposed method can also be used as a tool to investigate the validity of hadronic interaction models. In particular it has been shown that the intrinsic shower fluctuations are sometimes larger than the measured $X_{\max }$ dispersions. This happens in different energy intervals for the different models. At the highest energies, all models approach the lower boundary, and some of them enter the unphysical region, but the current systematic uncertainties prevent us from confidently rejecting any model. Provided that systematic uncertainties can be reduced in future data analysis, the method can be used to constrain 


\begin{tabular}{|c|c|c|c|c|}
\hline parameter & EPOS 1.99 & Sibyll 2.1 & QGSJet 01 & QGSJet II \\
\hline$X_{0}$ & $809.7 \pm 0.3$ & $795.1 \pm 0.3$ & $774.2 \pm 0.3$ & $781.8 \pm 0.3$ \\
\hline$D$ & $62.2 \pm 0.5$ & $57.7 \pm 0.5$ & $49.7 \pm 0.5$ & $45.8 \pm 0.5$ \\
\hline$\xi$ & $0.78 \pm 0.24$ & $-0.04 \pm 0.24$ & $-0.30 \pm 0.24$ & $-1.13 \pm 0.24$ \\
\hline$\delta$ & $0.08 \pm 0.21$ & $-0.04 \pm 0.21$ & $1.92 \pm 0.21$ & $1.71 \pm 0.21$ \\
\hline
\end{tabular}

Table 1. Parameters of formulae (2.6) and (2.7) for different hadronic interaction models setting $E_{0}$ $=10^{19} \mathrm{eV}$. The values are obtained fitting the mean $X_{\max }$ for showers generated for four different primaries H, He, $\mathrm{N}$ and Fe. Statistical error obtained from the fit are also given. All values are expressed in $\mathrm{g} \mathrm{cm}^{-2}$.

hadronic interaction models. The addition of new measurements, such as the muon content of EAS [32, 33], may allow us to place stronger bounds to the models.

\section{A Parameterization of the shower mean depth and its fluctuations}

The shower code chosen for this work is CONEX [15]. CONEX is a hybrid simulation code that is suited for fast one-dimensional simulations of shower profiles, including fluctuations. It combines Monte Carlo simulation of high energy interactions with a fast numerical solution of cascade equations for the resulting distributions of secondary particles. In our CONEX simulation we used the default energy thresholds settings of version v2r3.1. ${ }^{2}$

The parameters $X_{0}, D_{1}, \xi$ and $\delta$ used in equations (2.6) and (2.7) have been obtained by fitting CONEX showers for four different primaries $(\mathrm{H}, \mathrm{He}, \mathrm{N}$ and $\mathrm{Fe}$ ) in nine energy bins of width $\Delta \log _{10}(E / \mathrm{eV})=0.25$ ranging from $10^{18}$ to $10^{20} \mathrm{eV}$, and for all the hadronic models used in this work: EPOS 1.99 [11], Sibyll 2.1 [12], QGSJet 01 [13] and QGSJet II [14]. In total, about 25,000 showers have been used for each energy bin and for each hadronic model. The fit procedure always converges with mean (maximum) $\left\langle X_{\max }\right\rangle$ residuals from the simulated data of about $1(3) \mathrm{g} \mathrm{cm}^{-2}$ for all the models. The best fit values are reported in table 1 with their errors.

Shower variances have been fitted using the parameterization given in equations (2.8) and (2.9) and the same simulated data set described above. The mean (maximum) $\sigma\left(X_{\max }\right)$ residuals from the simulated data are about $1(3) \mathrm{g} \mathrm{cm}^{-2}$ for all the models. The best fit parameters are given in table 2 with their errors.

\section{Acknowledgments}

The successful installation, commissioning, and operation of the Pierre Auger Observatory would not have been possible without the strong commitment and effort from the technical and administrative staff in Malargüe.

We are very grateful to the following agencies and organizations for financial support: Comisión Nacional de Energía Atómica, Fundación Antorchas, Gobierno De La Provincia

\footnotetext{
${ }^{2}$ The hadron and muon cutoff (minimum energy) is $1 \mathrm{GeV}$, the cutoff for electrons, positrons and gammas (e/m particles) is $1 \mathrm{MeV}$, the threshold energy for solving cascade equations is $0.05,0.0005$ and 0.005 of the primary energy for hadrons, muons and e/m particles respectively. The above-threshold e/m interaction are simulated with the EGS4 program [34]. The low energy $(\mathrm{E}<80 \mathrm{GeV})$ interaction model is GEISHA [35].
} 


\begin{tabular}{|c|c|c|c|c|}
\hline parameter & EPOS 1.99 & Sibyll 2.1 & QGSJet 01 & QGSJet II \\
\hline$p_{0} \times\left(\mathrm{g}^{-2} \mathrm{~cm}^{4}\right)$ & $3279 \pm 51$ & $2785 \pm 46$ & $3852 \pm 55$ & $3163 \pm 49$ \\
\hline$p_{1} \times\left(\mathrm{g}^{-2} \mathrm{~cm}^{4}\right)$ & $-47 \pm 66$ & $-364 \pm 58$ & $-274 \pm 70$ & $-237 \pm 61$ \\
\hline$p_{2} \times\left(\mathrm{g}^{-2} \mathrm{~cm}^{4}\right)$ & $228 \pm 108$ & $152 \pm 93$ & $169 \pm 116$ & $60 \pm 100$ \\
\hline$a_{0}$ & $-0.461 \pm 0.006$ & $-0.368 \pm 0.008$ & $-0.451 \pm 0.006$ & $-0.386 \pm 0.007$ \\
\hline$a_{1}$ & $-0.0041 \pm 0.0016$ & $-0.0049 \pm 0.0023$ & $-0.0020 \pm 0.0016$ & $-0.0006 \pm 0.0021$ \\
\hline$b$ & $0.059 \pm 0.002$ & $0.039 \pm 0.002$ & $0.057 \pm 0.001$ & $0.043 \pm 0.002$ \\
\hline
\end{tabular}

Table 2. Parameters of formulae (2.8) and (2.9) for different hadronic interaction models setting $E_{0}$ $=10^{19} \mathrm{eV}$. The values are obtained fitting $\sigma^{2}\left(X_{\max }\right)$ for showers generated for four different primaries $\mathrm{H}, \mathrm{He}, \mathrm{N}$ and Fe. The statistical errors obtained from the fit are also given.

de Mendoza, Municipalidad de Malargüe, NDM Holdings and Valle Las Leñas, in gratitude for their continuing cooperation over land access, Argentina; the Australian Research Council; Conselho Nacional de Desenvolvimento Científico e Tecnológico (CNPq), Financiadora de Estudos e Projetos (FINEP), Fundação de Amparo à Pesquisa do Estado de Rio de Janeiro (FAPERJ), Fundação de Amparo à Pesquisa do Estado de São Paulo (FAPESP), Ministério de Ciência e Tecnologia (MCT), Brazil; AVCR AV0Z10100502 and AV0Z10100522, GAAV KJB100100904, MSMT-CR LA08016, LG11044, MEB111003, MSM0021620859, LA08015, TACR TA01010517 and GA U.K. 119810, Czech Republic; Centre de Calcul IN2P3/CNRS, Centre National de la Recherche Scientifique (CNRS), Conseil Régional Ile-de-France, Département Physique Nucléaire et Corpusculaire (PNCIN2P3/CNRS), Département Sciences de l'Univers (SDU-INSU/CNRS), France; Bundesministerium für Bildung und Forschung (BMBF), Deutsche Forschungsgemeinschaft (DFG), Finanzministerium Baden-Württemberg, Helmholtz-Gemeinschaft Deutscher Forschungszentren (HGF), Ministerium für Wissenschaft und Forschung, Nordrhein-Westfalen, Ministerium für Wissenschaft, Forschung und Kunst, Baden-Württemberg, Germany; Istituto Nazionale di Fisica Nucleare (INFN), Ministero dell'Istruzione, dell'Università e della Ricerca (MIUR), Italy; Consejo Nacional de Ciencia y Tecnología (CONACYT), Mexico; Ministerie van Onderwijs, Cultuur en Wetenschap, Nederlandse Organisatie voor Wetenschappelijk Onderzoek (NWO), Stichting voor Fundamenteel Onderzoek der Materie (FOM), Netherlands; Ministry of Science and Higher Education, Grant Nos. N N202 200239 and N N202 207238, Poland; Portuguese national funds and FEDER funds within COMPETE - Programa Operacional Factores de Competitividade through Fundação para a Ciência e a Tecnologia, Portugal; Romanian Authority for Scientific Research ANCS, CNDI-UEFISCDI partnership projects nr.20/2012 and nr.194/2012, project nr.1/ASPERA2/2012 ERA-NET and PN-II-RU-PD2011-3-0145-17, Romania; Ministry for Higher Education, Science, and Technology, Slovenian Research Agency, Slovenia; Comunidad de Madrid, FEDER funds, Ministerio de Ciencia e Innovación and Consolider-Ingenio 2010 (CPAN), Xunta de Galicia, Spain; The Leverhulme Foundation, Science and Technology Facilities Council, United Kingdom; Department of Energy, Contract Nos. DE-AC02-07CH11359, DE-FR02-04ER41300, DE-FG02-99ER41107, National Science Foundation, Grant No. 0450696, The Grainger Foundation U.S.A.; NAFOSTED, Vietnam; Marie Curie-IRSES/EPLANET, European Particle Physics Latin American Network, European Union 7th Framework Program, Grant No. PIRSES-2009-GA-246806; and UNESCO. 


\section{References}

[1] For a recent review see e.g. R. Engel, D. Heck and T. Pierog, Extensive air showers and hadronic interactions at high energy, Ann. Rev. Nucl. Part. Sci. 61 (2011) 467 [INSPIRE].

[2] See e.g. T.K. Gaisser, Cosmic Rays and Particle Physics, Cambridge University Press, Cambridge (1990).

[3] J. Linsley, Spectra, anisotropies and composition of cosmic rays above $1000 \mathrm{GeV}$, rapporteur paper in Proc. 18th International Cosmic Ray Conference (ICRC), Bangalore, India, 12 (1983) 135 [INSPIRE].

[4] J. Linsley, Proton-air and proton-proton cross sections from air shower data, in Proc. 19th International Cosmic Ray Conference (ICRC), San Diego, California, 6 (1985) 1 [INSPIRE].

[5] Pierre Auger collaboration, J. Abraham et al., Measurement of the Depth of Maximum of Extensive Air Showers above $10^{18}$ eV, Phys. Rev. Lett. 104 (2010) 091101 [arXiv:1002.0699] [INSPIRE].

[6] Pierre Auger collaboration, P. Facal San Luis, The distribution of shower maxima of UHECR air showers, in Proc. 32nd International Cosmic Ray Conference (ICRC), Beijing, China, 2 (2011) 225 [inSPIRE];

Pierre Auger collaboration, P. Abreu et al., The Pierre Auger Observatory II: Studies of Cosmic Ray Composition and Hadronic Interaction models, arXiv:1107.4804 [INSPIRE].

[7] See e.g. J. Matthews, A Heitler model of extensive air showers, Astropart. Phys. 22 (2005) 387 [INSPIRE] and references therein.

[8] J. Linsley, Structure of large air showers at depth $834 \mathrm{~g} / \mathrm{cm}^{2}$, in Proc. 15th International Cosmic Ray Conference (ICRC), Plovdiv, Bulgaria, 12 (1977) 89 [InSPIRE].

[9] T.K. Gaisser et al., Elongation Rate of Air Showers and Implications for $10^{17}-10^{18} \mathrm{eV}$ Particle Interactions, in Proc. 16th International Cosmic Ray Conference (ICRC), Kyoto, Japan, 9 (1979) 275 [INSPIRE].

[10] J. Linsley and A. Watson, Validity of scaling to $10^{20}-\mathrm{eV}$ and high-energy cosmic ray composition, Phys. Rev. Lett. 46 (1981) 459 [INSPIRE].

[11] T. Pierog and K. Werner, Muon Production in Extended Air Shower Simulations, Phys. Rev. Lett. 101 (2008) 171101 [astro-ph/0611311] [INSPIRE].

[12] E.-J. Ahn, R. Engel, T.K. Gaisser, P. Lipari and T. Stanev, Cosmic ray interaction event generator SIBYLL 2.1, Phys. Rev. D 80 (2009) 094003 [arXiv: 0906.4113] [INSPIRE].

[13] N. Kalmykov, S. Ostapchenko and A. Pavlov, Quark-gluon string model and EAS simulation problems at ultra-high energies, Nucl. Phys. Proc. Suppl. 52B (1997) 17 [InSPIRE].

[14] S. Ostapchenko, Non-linear screening effects in high energy hadronic interactions, Phys. Rev. D 74 (2006) 014026 [hep-ph/0505259] [INSPIRE].

[15] T. Pierog, M. Alekseeva, T. Bergmann, V. Chernatkin, R. Engel, et al., First results of fast one-dimensional hybrid simulation of EAS using CONEX, Nucl. Phys. Proc. Suppl. 151 (2006) 159 [astro-ph/0411260] [INSPIRE].

[16] HIRES collaboration, D. Bird et al., Evidence for correlated changes in the spectrum and composition of cosmic rays at extremely high-energies, Phys. Rev. Lett. 71 (1993) 3401 [INSPIRE].

[17] High Resolution Fly's Eye collaboration, R. Abbasi, et al., A Study of the composition of ultrahigh energy cosmic rays using the High Resolution Fly's Eye, Astrophys. J. 622 (2005) 910 [astro-ph/0407622] [INSPIRE].

[18] HiRes collaboration, R. Abbasi et al., Indications of Proton-Dominated Cosmic Ray Composition above 1.6 EeV, Phys. Rev. Lett. 104 (2010) 161101 [arXiv:0910.4184] [INSPIRE].

[19] S. Knurenko and A. Sabourov, Spectrum and mass composition of cosmic rays in the energy range $10^{15}-10^{18} \mathrm{eV}$ derived from the Yakutsk array data, in Proc. 32nd International Cosmic Ray Conference (ICRC), Beijing, China, 1 (2011) 189, [arXiv:1112.2430] [InSPIRE]. 
[20] Telescope Array collaboration, C.C. Jui, Cosmic Ray in the Northern Hemisphere: Results from the Telescope Array Experiment, Proc. APS DPF Meeting, (2011) Providence, RI, U.S.A., J. Phys. Conf. Ser. 404 (2012) 012037 [arXiv:1110.0133] [InSPIRE].

[21] R. Walker and A. Watson, Measurement of the fluctuations in the depth of maximum of showers produced by primary particles of energy greater than $1.5 \times 10^{17} \mathrm{eV}$, J. Phys. G 8 (1982) 1131 [INSPIRE].

[22] Pierre Auger collaboration, D. Garcia-Pinto, Measurements of the Longitudinal Development of Air Showers with the Pierre Auger Observatory, in Proc. 32nd International Cosmic Ray Conference (ICRC), Beijing, China, 2 (2011) 87 [InSPIRE];

PIERre Auger collaboration, P. Abreu et al., The Pierre Auger Observatory II: Studies of Cosmic Ray Composition and Hadronic Interaction models, arXiv: 1107.4804 [INSPIRE]; http://www.auger.org/technical_info/ICRC2011/shower_development_data.txt.

[23] M. Unger, EAS Studies of Cosmic Rays above $10^{16} \mathrm{eV}$, rapporteur paper in Proc. 32nd International Cosmic Ray Conference (ICRC), Beijing, China, 12 (2011) 225.

[24] L. Cazon and R. Ulrich, The non-linearity between $\langle\ln A\rangle$ and $\left\langle X_{\max }\right\rangle$ induced by the acceptance of fluorescence telescopes, Astropart. Phys. 38 (2012) 41 [arXiv:1203.1781] [INSPIRE].

[25] Pierre Auger collaboration, R. Pesce, Energy calibration of data recorded with the surface detectors of the Pierre Auger Observatory: an update, in Proc. 32nd International Cosmic Ray Conference (ICRC), Beijing, China, 2 (2011) 214 [INSPIRE];

Pierre Auger collaboration, P. Abreu et al., The Pierre Auger Observatory I: The Cosmic Ray Energy Spectrum and Related Measurements, arXiv:1107.4809 [INSPIRE].

[26] Pierre Auger collaboration, P. Abreu et al., Measurement of the proton-air cross-section at $\sqrt{s}=57$ TeV with the Pierre Auger Observatory, Phys. Rev. Lett. 109 (2012) 062002 [arXiv:1208.1520] [INSPIRE].

[27] A. Watson and J. Wilson, Fluctuation studies of large air showers - the composition of primary cosmic ray particles of energy $E_{p} \sim 10^{18} \mathrm{eV}, \mathrm{J}$. Phys. A 7 (1974) 1199 [InSPIRE].

[28] R. Walker and A. Watson, Measurement of the elongation rate of extensive air showers produced by primary cosmic rays of energy above $2 \times 10^{17} \mathrm{eV}$, J. Phys. G 7 (1981) 1297 [INSPIRE].

[29] K.-H. Kampert and M. Unger, Measurements of the Cosmic Ray Composition with Air Shower Experiments, Astropart. Phys. 35 (2012) 660 [arXiv:1201.0018] [InSPIRE].

[30] A.M. Taylor, M. Ahlers and F.A. Aharonian, The need for a local source of UHE CR nuclei, Phys. Rev. D 84 (2011) 105007 [arXiv:1107.2055] [INSPIRE].

[31] D. Allard, Extragalactic propagation of ultrahigh energy cosmic-rays, Astropart. Phys. 39-40 (2012) 33 [arXiv:1111.3290] [INSPIRE].

[32] PIERre Auger collaboration, J. Allen, Interpretation of the signals produced by showers from cosmic rays of $10^{19} \mathrm{eV}$ observed in the surface detectors of the Pierre Auger Observatory, in Proc. 32nd International Cosmic Ray Conference (ICRC), Beijing, China, 2 (2011) 83 [INSPIRE];

PIERre Auger collaboration, P. Abreu et al., The Pierre Auger Observatory II: Studies of Cosmic Ray Composition and Hadronic Interaction models, arXiv:1107.4804 [INSPIRE].

[33] Pierre Auger collaboration, G. Rodriguez, Reconstruction of inclined showers at the Pierre Auger Observatory: implications for the muon contenty, in Proc. 32nd International Cosmic Ray Conference (ICRC), Beijing, China, 2 (2011) 95 [INSPIRE];

Pierre Auger collaboration, P. Abreu et al., The Pierre Auger Observatory I: The Cosmic Ray Energy Spectrum and Related Measurements, arXiv:1107.4809 [INSPIRE].

[34] W. Nelson et al., The Egs4 Code System, SLAC-265, Stanford Linear Accelerator Center (1985).

[35] GEISHA, H. Fesefeldt, RWTH Aachen report PITHA 85/2 (1985). 


\section{The Pierre Auger collaboration}

P. Abreu ${ }^{61}$, M. Aglietta ${ }^{49}$, M. Ahlers ${ }^{90}$, E.J. Ahn ${ }^{78}$, I.F.M. Albuquerque ${ }^{15}$, I. Allekotte ${ }^{1}$, J. Allen ${ }^{82}$, P. Allison ${ }^{84}$, A. Almela ${ }^{11,7}$, J. Alvarez Castillo ${ }^{54}$, J. Alvarez-Muñiz ${ }^{71}$, R. Alves Batista ${ }^{16}$, M. Ambrosio ${ }^{43}$, A. Aminaei ${ }^{55}$, L. Anchordoqui ${ }^{91}$, S. Andringa ${ }^{61}$, T. Antičić ${ }^{22}$, C. Aramo ${ }^{43}$, F. Arqueros ${ }^{68}$, H. Asorey ${ }^{1}$, P. Assis ${ }^{61}$, J. Aublin ${ }^{28}$, M. Ave ${ }^{71}$, M. Avenier ${ }^{29}$, G. Avila ${ }^{10}$, A.M. Badescu ${ }^{64}$, K.B. Barber ${ }^{12}$, A.F. Barbosa ${ }^{13} \ddagger$, R. Bardenet ${ }^{27}$, B. Baughman ${ }^{84 ~ c}$, J. Bäuml ${ }^{33}$, C. Baus $^{35}$, J.J. Beatty ${ }^{84}$, K.H. Becker ${ }^{32}$, A. Bellétoile ${ }^{31}$, J.A. Bellido ${ }^{12}$, S. BenZvi ${ }^{90}$, C. Berat ${ }^{29}$, X. Bertou ${ }^{1}$, P.L. Biermann ${ }^{36}$, P. Billoir ${ }^{28}$, F. Blanco ${ }^{68}$, M. Blanco ${ }^{28}$, C. Bleve ${ }^{32}$, H. Blümer ${ }^{35}, 33$, M. Boháčová ${ }^{24}$, D. Boncioli ${ }^{44}$, C. Bonifazi ${ }^{20}$, R. Bonino ${ }^{49}$, N. Borodai ${ }^{59}$, J. Brack ${ }^{76}$, I. Brancus $^{62}$, P. Brogueira ${ }^{61}$, W.C. Brown ${ }^{77}$, P. Buchholz ${ }^{39}$, A. Bueno ${ }^{70}$, L. Buroker ${ }^{91}$, R.E. Burton ${ }^{74}$, M. Buscemi ${ }^{43}$, K.S. Caballero-Mora ${ }^{71,85}$, B. Caccianiga ${ }^{42}$, L. Caccianiga ${ }^{28}$, L. Caramete $^{36}$, R. Caruso ${ }^{45}$, A. Castellina ${ }^{49}$, G. Cataldi ${ }^{47}$, L. Cazon ${ }^{61}$, R. Cester ${ }^{46}$, S.H. Cheng ${ }^{85}$, A. Chiavassa ${ }^{49}$, J.A. Chinellato ${ }^{16}$, J. Chudoba ${ }^{24}$, M. Cilmo ${ }^{43}$, R.W. Clay $^{12}$, G. Cocciolo ${ }^{47}$, R. Colalillo ${ }^{43}$, L. Collica ${ }^{42}$, M.R. Coluccia ${ }^{47}$, R. Conceição ${ }^{61}$, F. Contreras $^{9}$, H. Cook ${ }^{72}$, M.J. Cooper $^{12}$, J. Coppens ${ }^{55,57}$, S. Coutu ${ }^{85}$, C.E. Covault ${ }^{74}$, A. Criss ${ }^{85}$, J. Cronin ${ }^{86}$, A. Curutiu ${ }^{36}$, R. Dallier ${ }^{31,30}$, B. Daniel ${ }^{16}$, S. Dasso ${ }^{5,3}$, K. Daumiller ${ }^{33}$, B.R. Dawson ${ }^{12}$, R.M. de Almeida ${ }^{21}$, M. De Domenico ${ }^{45}$, S.J. de Jong ${ }^{55,57}$, G. De La Vega ${ }^{8}$, W.J.M. de Mello Junior ${ }^{16}$, J.R.T. de Mello Neto ${ }^{20}$, I. De Mitri ${ }^{47}$, V. de Souza ${ }^{14}$, K.D. de Vries ${ }^{56}$, L. del Peral ${ }^{69}$, O. Deligny ${ }^{26}$, H. Dembinski ${ }^{33}$, N. Dhital ${ }^{81}$, C. Di Giulio $^{44}$, J.C. Diaz ${ }^{81}$, M.L. Díaz Castro ${ }^{13}$, P.N. Diep ${ }^{92}$, F. Diogo ${ }^{61}$, C. Dobrigkeit ${ }^{16}$, W. Docters ${ }^{56}$, J.C. D'Olivo ${ }^{54}$, P.N. Dong ${ }^{92,26}$, A. Dorofeev ${ }^{76}$, J.C. dos Anjos ${ }^{13}$, M.T. Dova ${ }^{4}$, D. D'Urso ${ }^{43}$, J. Ebr ${ }^{24}$, R. Engel ${ }^{33}$, M. Erdmann ${ }^{37}$, C.O. Escobar ${ }^{78,16}$, J. Espadanal ${ }^{61}$, A. Etchegoyen ${ }^{7,11}$ P. Facal San Luis ${ }^{86}$, H. Falcke ${ }^{55,58,57}$, K. Fang ${ }^{86}$, G. Farrar ${ }^{82}$, A.C. Fauth ${ }^{16}$, N. Fazzini ${ }^{78}$,

A.P. Ferguson ${ }^{74}$, B. Fick ${ }^{81}$, J.M. Figueira ${ }^{7}$, A. Filevich ${ }^{7}$, A. Filipčič ${ }^{65}$, 66, S. Fliescher ${ }^{37}$, B.D. Fox ${ }^{87}$, C.E. Fracchiolla ${ }^{76}$, E.D. Fraenkel ${ }^{56}$, O. Fratu ${ }^{64}$, U. Fröhlich ${ }^{39}$, B. Fuchs ${ }^{35}$, R. Gaior ${ }^{28}$,

R.F. Gamarra ${ }^{7}$, S. Gambetta ${ }^{40}$, B. García ${ }^{8}$, S.T. Garcia Roca ${ }^{71}$, D. Garcia-Gamez ${ }^{27}$,

D. Garcia-Pinto ${ }^{68}$, G. Garilli ${ }^{45}$, A. Gascon Bravo ${ }^{70}$, H. Gemmeke ${ }^{34}$, P.L. Ghia ${ }^{28}$, M. Giller ${ }^{60}$, J. Gitto $^{8}$, C. Glaser ${ }^{37}$, H. Glass ${ }^{78}$, G. Golup ${ }^{1}$, F. Gomez Albarracin ${ }^{4}$, M. Gómez Berisso ${ }^{1}$, P.F. Gómez Vitale ${ }^{10}$, P. Gonçalves ${ }^{61}$, J.G. Gonzalez ${ }^{35}$, B. Gookin ${ }^{76}$, A. Gorgi ${ }^{49}$, P. Gorham ${ }^{87}$, P. Gouffon ${ }^{15}$, S. Grebe ${ }^{55,57}$, N. Griffith ${ }^{84}$, A.F. Grillo ${ }^{50}$, T.D. Grubb ${ }^{12}$, Y. Guardincerri ${ }^{3}$, F. Guarino ${ }^{43}$, G.P. Guedes ${ }^{17}$, P. Hansen ${ }^{4}$, D. Harari ${ }^{1}$, T.A. Harrison ${ }^{12}$, J.L. Harton ${ }^{76}$, A. Haungs ${ }^{33}$, T. Hebbeker ${ }^{37}$, D. Heck ${ }^{33}$, A.E. Herve ${ }^{12}$, G.C. Hill ${ }^{12}$, C. Hojvat ${ }^{78}$, N. Hollon ${ }^{86}$, V.C. Holmes ${ }^{12}$, P. Homola ${ }^{59}$, J.R. Hörandel ${ }^{55,57}$, P. Horvath ${ }^{25}$, M. Hrabovský ${ }^{25,24}$, D. Huber ${ }^{35}$, T. Huege ${ }^{33}$, A. Insolia ${ }^{45}$, F. Ionita ${ }^{86}$, S. Jansen ${ }^{55,57}$, C. Jarne ${ }^{4}$, S. Jiraskova ${ }^{55}$, M. Josebachuili ${ }^{7}$, K. Kadija ${ }^{22}$, K.H. Kampert ${ }^{32}$, P. Karhan ${ }^{23}$, P. Kasper ${ }^{78}$, I. Katkov ${ }^{35}$, B. Kégl ${ }^{27}$, B. Keilhauer ${ }^{33}$, A. Keivani ${ }^{80}$, J.L. Kelley ${ }^{55}$, E. Kemp ${ }^{16}$, R.M. Kieckhafer ${ }^{81}$, H.O. Klages ${ }^{33}$, M. Kleifges ${ }^{34}$, J. Kleinfeller ${ }^{9,33}$, J. Knapp ${ }^{72}$, K. Kotera ${ }^{86}$, R. Krause ${ }^{37}$, N. Krohm² ${ }^{32}$ O. Krömer ${ }^{34}$, D. Kruppke-Hansen ${ }^{32}$,

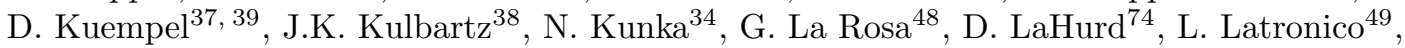
R. Lauer ${ }^{89}$, M. Lauscher ${ }^{37}$, P. Lautridou ${ }^{31}$, S. Le $\mathrm{Coz}^{29}$, M.S.A.B. Leão ${ }^{19}$, D. Lebrun ${ }^{29}$, P. Lebrun ${ }^{78}$, M.A. Leigui de Oliveira ${ }^{19}$, A. Letessier-Selvon ${ }^{28}$, I. Lhenry-Yvon ${ }^{26}$, K. Link ${ }^{35}$, R. López ${ }^{51}$, A. Lopez Agüera ${ }^{71}$, K. Louedec ${ }^{29,} 27$, J. Lozano Bahilo ${ }^{70}$, L. Lu ${ }^{72}$, A. Lucero ${ }^{7}$, M. Ludwig ${ }^{35}$, H. Lyberis ${ }^{20,26}$, M.C. Maccarone ${ }^{48}$, C. Macolino ${ }^{28}$, M. Malacari ${ }^{12}$, S. Maldera ${ }^{49}$, J. Maller ${ }^{31}$, D. Mandat ${ }^{24}$,

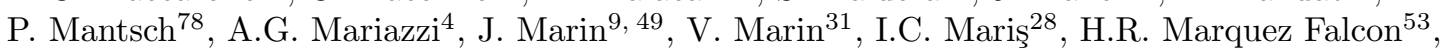
G. Marsella ${ }^{47}$, D. Martello ${ }^{47}$, L. Martin ${ }^{31,30}$, H. Martinez ${ }^{52}$, O. Martínez Bravo ${ }^{51}$, D. Martraire ${ }^{26}$, J.J. Masías Meza ${ }^{3}$, H.J. Mathes ${ }^{33}$, J. Matthews ${ }^{80}$, J.A.J. Matthews ${ }^{89}$, G. Matthiae ${ }^{44}$, D. Maurel ${ }^{33}$, D. Maurizio ${ }^{13,46}$, E. Mayotte ${ }^{75}$, P.O. Mazur ${ }^{78}$, G. Medina-Tanco ${ }^{54}$, M. Melissas ${ }^{35}$, D. Melo ${ }^{7}$, E. Menichetti ${ }^{46}$, A. Menshikov ${ }^{34}$, S. Messina ${ }^{56}$, R. Meyhandan ${ }^{87}$, S. Mićanović ${ }^{22}$, M.I. Micheletti ${ }^{6}$, L. Middendorf ${ }^{37}$, I.A. Minaya ${ }^{68}$, L. Miramonti ${ }^{42}$, B. Mitrica ${ }^{62}$, L. Molina-Bueno ${ }^{70}$, S. Mollerach $^{1}$, M. Monasor ${ }^{86}$, D. Monnier Ragaigne ${ }^{27}$, F. Montanet $^{29}$, B. Morales ${ }^{54}$, C. Morello ${ }^{49}$, J.C. Moreno ${ }^{4}$, M. Mostafá ${ }^{76}$, C.A. Moura ${ }^{19}$, M.A. Muller ${ }^{16}$, G. Müller ${ }^{37}$, M. Münchmeyer ${ }^{28}$, R. Mussa ${ }^{46}$,

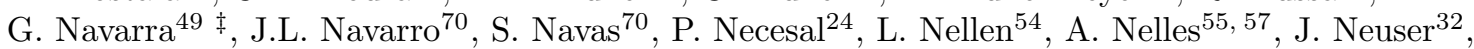
P.T. Nhung ${ }^{92}$, M. Niechciol ${ }^{39}$, L. Niemietz ${ }^{32}$, N. Nierstenhoefer ${ }^{32}$, T. Niggemann ${ }^{37}$, D. Nitz ${ }^{81}$,




D. Pakk Selmi-Dei ${ }^{16}$, M. Palatka ${ }^{24}$, J. Pallotta ${ }^{2}$, N. Palmieri ${ }^{35}$, G. Parente ${ }^{71}$, A. Parra ${ }^{71}$,

S. Pastor ${ }^{67}$, T. Paul ${ }^{91,83}$, M. Pech ${ }^{24}$, J. Pȩkala ${ }^{59}$, R. Pelayo ${ }^{51,}$ 71, I.M. Pepe ${ }^{18}$, L. Perrone ${ }^{47}$,

R. Pesce $^{40}$, E. Petermann ${ }^{88}$, S. Petrera ${ }^{41}$, A. Petrolini ${ }^{40}$, Y. Petrov ${ }^{76}$, C. Pfendner ${ }^{90}$, R. Piegaia ${ }^{3}$,

T. Pierog ${ }^{33}$, P. Pieroni ${ }^{3}$, M. Pimenta ${ }^{61}$, V. Pirronello ${ }^{45}$, M. Platino ${ }^{7}$, M. Plum ${ }^{37}$, V.H. Ponce ${ }^{1}$,

M. Pontz ${ }^{39}$, A. Porcelli ${ }^{33}$, P. Privitera ${ }^{86}$, M. Prouza ${ }^{24},{\text { E.J. } \text { Quel }^{2} \text {, S. Querchfeld }}^{32}$, J. Rautenberg ${ }^{32}$,

O. Ravel ${ }^{31}$, D. Ravignani ${ }^{7}$, B. Revenu ${ }^{31}$, J. Ridky ${ }^{24}$, S. Riggi ${ }^{48,71}$, M. Risse ${ }^{39}$, P. Ristori ${ }^{2}$,

H. Rivera ${ }^{42}$, V. Rizi ${ }^{41}$, J. Roberts ${ }^{82}$, W. Rodrigues de Carvalho ${ }^{71}$, I. Rodriguez Cabo ${ }^{71}$,

G. Rodriguez Fernandez ${ }^{44,71}$, J. Rodriguez Martino ${ }^{9}$, J. Rodriguez Rojo ${ }^{9}$, M.D. Rodríguez-Frías ${ }^{69}$,

G. $\operatorname{Ros}^{69}$, J. Rosado ${ }^{68}$, T. Rossler ${ }^{25}$, M. Roth ${ }^{33}$, B. Rouillé-d'Orfeuil ${ }^{86}$, E. Roulet $^{1}$, A.C. Rovero ${ }^{5}$,

C. Rühle ${ }^{34}$, S.J. Saffi ${ }^{12}$, A. Saftoiu ${ }^{62}$, F. Salamida ${ }^{26}$, H. Salazar ${ }^{51}$, F. Salesa Greus ${ }^{76}$, G. Salina ${ }^{44}$,

F. Sánchez ${ }^{7}$, C.E. Santo ${ }^{61}$, E. Santos ${ }^{61}$, E.M. Santos ${ }^{20}$, F. Sarazin ${ }^{75}$, B. Sarkar ${ }^{32}$, R. Sato ${ }^{9}$,

N. Scharf ${ }^{37}$, V. Scherini ${ }^{42}$, H. Schieler ${ }^{33}$, P. Schiffer ${ }^{38}$, A. Schmidt ${ }^{34}$, O. Scholten ${ }^{56}$,

H. Schoorlemmer ${ }^{55}$, 57 , J. Schovancova ${ }^{24}$, P. Schovánek ${ }^{24}$, F. Schröder ${ }^{33}$, J. Schulz ${ }^{55}$, D. Schuster ${ }^{75}$, S.J. Sciutto ${ }^{4}$, M. Scuderi ${ }^{45}$, A. Segreto ${ }^{48}$, M. Settimo ${ }^{39,47}$, A. Shadkam ${ }^{80}$, R.C. Shellard ${ }^{13}$,

I. Sidelnik ${ }^{1}$, G. Sigl ${ }^{38}$, O. Sima ${ }^{63}$, A. Śmiałkowski ${ }^{60}$, R. Šmída ${ }^{33}$, G.R. Snow ${ }^{88}$, P. Sommers ${ }^{85}$,

J. Sorokin ${ }^{12}$, H. Spinka ${ }^{73,78}$, R. Squartini ${ }^{9}$, Y.N. Srivastava ${ }^{83}$, S. Stanič ${ }^{66}$, J. Stapleton ${ }^{84}$,

J. Stasielak ${ }^{59}$, M. Stephan ${ }^{37}$, M. Straub ${ }^{37}$, A. Stutz ${ }^{29}$, F. Suarez ${ }^{7}$, T. Suomijärvi ${ }^{26}$,

A.D. Supanitsky ${ }^{5}$, T. Šuša ${ }^{22}$, M.S. Sutherland ${ }^{80}$, J. Swain ${ }^{83}$, Z. Szadkowski ${ }^{60}$, M. Szuba ${ }^{33}$,



C. Timmermans ${ }^{57}, 55$, W. Tkaczyk ${ }^{60} \ddagger$ C.J. Todero Peixoto ${ }^{14}$, G. Toma ${ }^{62}$, L. Tomankova ${ }^{24}$,

B. Tomé ${ }^{61}$, A. Tonachini ${ }^{46}$, G. Torralba Elipe ${ }^{71}$, D. Torres Machado ${ }^{31}$, P. Travnicek ${ }^{24}$,

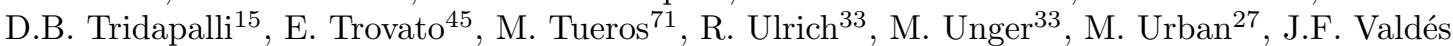

Galicia $^{54}$, I. Valiño ${ }^{71}$, L. Valore ${ }^{43}$, G. van $\mathrm{Aar}^{55}$. A.M. van den Berg ${ }^{56}$, S. van Velzen ${ }^{55}$, A. van

Vliet $^{38}$, E. Varela ${ }^{51}$, B. Vargas Cárdenas ${ }^{54}$, G. Varner ${ }^{87}$, J.R. Vázquez ${ }^{68}$, R.A. Vázquez ${ }^{71}$,

D. Veberič ${ }^{66,65}$, V. Verzi ${ }^{44}$, J. Vicha ${ }^{24}$, M. Videla ${ }^{8}$, L. Villaseñor ${ }^{53}$, H. Wahlberg ${ }^{4}$, P. Wahrlich ${ }^{12}$,

O. Wainberg ${ }^{7,11}$, D. Walz ${ }^{37}$, A.A. Watson ${ }^{72}$, M. Weber ${ }^{34}$, K. Weidenhaupt ${ }^{37}$, A. Weindl ${ }^{33}$,

F. Werner ${ }^{33}$, S. Westerhoff ${ }^{90}$, B.J. Whelan ${ }^{85}$, A. Widom ${ }^{83}$, G. Wieczorek ${ }^{60}$, L. Wiencke ${ }^{75}$,

B. Wilczyńska ${ }^{59} \ddagger$ H. Wilczyńskii ${ }^{59}$, M. Will ${ }^{33}$, C. Williams ${ }^{86}$, T. Winchen ${ }^{37}$, M. Wommer ${ }^{33}$,



B. Zamorano Garcia ${ }^{70}$, E. Zas ${ }^{71}$, D. Zavrtanik ${ }^{66,65}$, M. Zavrtanik ${ }^{65,66}$, I. Zaw ${ }^{82} d$, A. Zepeda ${ }^{52} b$,

J. Zhou ${ }^{86}$, Y. Zhu ${ }^{34}$, M. Zimbres Silva ${ }^{32,16}$, M. Ziolkowski ${ }^{39}$

${ }^{1}$ Centro Atómico Bariloche and Instituto Balseiro (CNEA-UNCuyo-CONICET), San Carlos de Bariloche,

Argentina

2 Centro de Investigaciones en Láseres y Aplicaciones, CITEDEF and CONICET, Argentina

${ }^{3}$ Departamento de Física, FCEyN, Universidad de Buenos Aires y CONICET, Argentina

${ }^{4}$ IFLP, Universidad Nacional de La Plata and CONICET, La Plata, Argentina

${ }^{5}$ Instituto de Astronomía y Fúsica del Espacio (CONICET-UBA), Buenos Aires, Argentina

${ }^{6}$ Instituto de Física de Rosario (IFIR) - CONICET/U.N.R. and Facultad de Ciencias Bioquímicas y

Farmacéuticas U.N.R., Rosario, Argentina

7 Instituto de Tecnologías en Detección y Astropartículas (CNEA, CONICET, UNSAM), Buenos Aires,

Argentina

${ }^{8}$ National Technological University, Faculty Mendoza (CONICET/CNEA), Mendoza, Argentina

${ }^{9}$ Observatorio Pierre Auger, Malargüe, Argentina

${ }^{10}$ Observatorio Pierre Auger and Comisión Nacional de Energía Atómica, Malargüe, Argentina

11 Universidad Tecnológica Nacional - Facultad Regional Buenos Aires, Buenos Aires, Argentina

12 University of Adelaide, Adelaide, S.A., Australia

13 Centro Brasileiro de Pesquisas Fisicas, Rio de Janeiro, RJ, Brazil

${ }^{14}$ Universidade de São Paulo, Instituto de Física, São Carlos, SP, Brazil

15 Universidade de São Paulo, Instituto de Física, São Paulo, SP, Brazil

16 Universidade Estadual de Campinas, IFGW, Campinas, SP, Brazil

17 Universidade Estadual de Feira de Santana, Brazil

18 Universidade Federal da Bahia, Salvador, BA, Brazil

19 Universidade Federal do ABC, Santo André, SP, Brazil

${ }^{20}$ Universidade Federal do Rio de Janeiro, Instituto de Física, Rio de Janeiro, RJ, Brazil 
${ }^{21}$ Universidade Federal Fluminense, EEIMVR, Volta Redonda, RJ, Brazil

${ }^{22}$ Rudjer Bošković Institute, 10000 Zagreb, Croatia

${ }^{23}$ Charles University, Faculty of Mathematics and Physics, Institute of Particle and Nuclear Physics,

Prague, Czech Republic

${ }^{24}$ Institute of Physics of the Academy of Sciences of the Czech Republic, Prague, Czech Republic

${ }^{25}$ Palacky University, RCPTM, Olomouc, Czech Republic

${ }^{26}$ Institut de Physique Nucléaire d'Orsay (IPNO), Université Paris 11, CNRS-IN2P3, Orsay, France

${ }^{27}$ Laboratoire de l'Accélérateur Linéaire (LAL), Université Paris 11, CNRS-IN2P3, France

28 Laboratoire de Physique Nucléaire et de Hautes Energies (LPNHE), Universités Paris 6 et Paris 7 ,

CNRS-IN2P3, Paris, France

${ }^{29}$ Laboratoire de Physique Subatomique et de Cosmologie (LPSC), Université Joseph Fourier Grenoble, CNRS-IN2P3, Grenoble INP, France

30 Station de Radioastronomie de Nançay, Observatoire de Paris, CNRS/INSU, France

31 SUBATECH, École des Mines de Nantes, CNRS-IN2P3, Université de Nantes, France

32 Bergische Universität Wuppertal, Wuppertal, Germany

${ }^{33}$ Karlsruhe Institute of Technology - Campus North - Institut für Kernphysik, Karlsruhe, Germany

${ }^{34}$ Karlsruhe Institute of Technology - Campus North - Institut für Prozessdatenverarbeitung und Elektronik, Karlsruhe, Germany

${ }^{35}$ Karlsruhe Institute of Technology - Campus South - Institut für Experimentelle Kernphysik (IEKP),

Karlsruhe, Germany

${ }^{36}$ Max-Planck-Institut für Radioastronomie, Bonn, Germany

37 RWTH Aachen University, III. Physikalisches Institut A, Aachen, Germany

38 Universität Hamburg, Hamburg, Germany

39 Universität Siegen, Siegen, Germany

40 Dipartimento di Fisica dell'Università and INFN, Genova, Italy

41 Università dell'Aquila and INFN, L'Aquila, Italy

42 Università di Milano and Sezione INFN, Milan, Italy

43 Università di Napoli "Federico II" and Sezione INFN, Napoli, Italy

44 Università di Roma II "Tor Vergata" and Sezione INFN, Roma, Italy

45 Università di Catania and Sezione INFN, Catania, Italy

46 Università di Torino and Sezione INFN, Torino, Italy

47 Dipartimento di Matematica e Fisica "E. De Giorgi" dell'Università del Salento and Sezione INFN, Lecce, Italy

48 Istituto di Astrofisica Spaziale e Fisica Cosmica di Palermo (INAF), Palermo, Italy

${ }^{49}$ Istituto di Fisica dello Spazio Interplanetario (INAF), Università di Torino and Sezione INFN, Torino, Italy

50 INFN, Laboratori Nazionali del Gran Sasso, Assergi (L'Aquila), Italy

51 Benemérita Universidad Autónoma de Puebla, Puebla, Mexico

52 Centro de Investigación y de Estudios Avanzados del IPN (CINVESTAV), México, Mexico

${ }^{53}$ Universidad Michoacana de San Nicolas de Hidalgo, Morelia, Michoacan, Mexico

54 Universidad Nacional Autonoma de Mexico, Mexico, D.F., Mexico

${ }^{55}$ IMAPP, Radboud University Nijmegen, Netherlands

${ }^{56}$ Kernfysisch Versneller Instituut, University of Groningen, Groningen, Netherlands

57 Nikhef, Science Park, Amsterdam, Netherlands

58 ASTRON, Dwingeloo, Netherlands

59 Institute of Nuclear Physics PAN, Krakow, Poland

60 University of Eódź, Eódź, Poland

${ }^{61}$ LIP and Instituto Superior Técnico, Technical University of Lisbon, Portugal

62 'Horia Hulubei' National Institute for Physics and Nuclear Engineering, Bucharest- Magurele, Romania

63 University of Bucharest, Physics Department, Romania

64 University Politehnica of Bucharest, Romania

65 J. Stefan Institute, Ljubljana, Slovenia

${ }^{66}$ Laboratory for Astroparticle Physics, University of Nova Gorica, Slovenia

67 Institut de Física Corpuscular, CSIC-Universitat de València, Valencia, Spain

68 Universidad Complutense de Madrid, Madrid, Spain

69 Universidad de Alcalá, Alcalá de Henares (Madrid), Spain

${ }^{70}$ Universidad de Granada and C.A.F.P.E., Granada, Spain

${ }^{71}$ Universidad de Santiago de Compostela, Spain 
72 School of Physics and Astronomy, University of Leeds, United Kingdom

73 Argonne National Laboratory, Argonne, IL, U.S.A.

${ }^{74}$ Case Western Reserve University, Cleveland, OH, U.S.A.

75 Colorado School of Mines, Golden, CO, U.S.A.

${ }^{76}$ Colorado State University, Fort Collins, CO, U.S.A.

77 Colorado State University, Pueblo, CO, U.S.A.

78 Fermilab, Batavia, IL, U.S.A.

${ }^{79}$ Los Alamos National Laboratory, Los Alamos, NM, U.S.A.

${ }^{80}$ Louisiana State University, Baton Rouge, LA, U.S.A.

81 Michigan Technological University, Houghton, MI, U.S.A.

${ }^{82}$ New York University, New York, NY, U.S.A.

${ }^{83}$ Northeastern University, Boston, MA, U.S.A.

${ }^{84}$ Ohio State University, Columbus, OH, U.S.A.

85 Pennsylvania State University, University Park, PA, U.S.A.

${ }^{86}$ University of Chicago, Enrico Fermi Institute, Chicago, IL, U.S.A.

87 University of Hawaii, Honolulu, HI, U.S.A.

88 University of Nebraska, Lincoln, NE, U.S.A.

${ }^{89}$ University of New Mexico, Albuquerque, NM, U.S.A.

${ }^{90}$ University of Wisconsin, Madison, WI, U.S.A.

91 University of Wisconsin, Milwaukee, WI, U.S.A.

${ }^{92}$ Institute for Nuclear Science and Technology (INST), Hanoi, Vietnam

( $\ddagger$ Deceased

(a) Now at Konan University

(b) Also at the Universidad Autonoma de Chiapas on leave of absence from Cinvestav

(c) Now at University of Maryland

(d) Now at NYU Abu Dhabi 